\title{
Analysis of mRNA abundance and stability by ribonuclease protection assay
}

\author{
Cristina Romero-López ${ }^{1}$, Alicia Barroso-delJesus ${ }^{1}$, Pablo Menendez ${ }^{2 *}$ and \\ Alfredo Berzal-Herranz $^{1 *}$
}

${ }^{1}$ Instituto de Parasitología y Biomedicina "López-Neyra”, CSIC, Parque Tecnológico de Ciencias de la Salud, Av. del Conocimiento s/n, Armilla 18100, Granada, Spain. ${ }^{2}$ Andalusian Stem Cell Bank, Centro de Investigación Biomédica, Parque Tecnológico de Ciencias de la Salud, Av. del Conocimiento, Armilla 18100, Granada, Spain.

* To whom correspondence should be addressed:

Pablo Menéndez: Andalusian Stem Cell Bank, Centro de Investigación Biomédica, Parque Tecnológico de Ciencias de la Salud, Av. del Conocimiento s/n, Armilla 18100, Granada, Spain. E-mail: pablo.menendez@juntadeandalucia.es. Phone: +34 958894 672. Fax: +34 958 894652.

Alfredo Berzal-Herranz: Instituto de Parasitología y Biomedicina "López-Neyra", CSIC, Parque Tecnológico de Ciencias de la Salud, Av. del Conocimiento s/n, Armilla 18100, Granada, Spain. E-mail: aberzalh@ipb.csic.es. Phone: +34 958 181 648. Fax: +34 958181 632

\begin{abstract}
Gene expression is a multi-step process which proceeds from DNA through RNA to protein. The tight regulation of this process is essential for overall cellular integrity and physiological homeostasis. Regulation of the messenger RNA (mRNA) levels has emerged as a crucial event in the modulation of the expression of genetic information. The mechanisms by which this process occurs have been extensively studied and begin to be much better understood. They involve a network of complex pathways that use intrinsic features of the target mRNA, like stability, to control its relative abundance in the cytoplasm. Thus, the analysis of the mRNA stability and abundance is essential to properly undertake gene expression studies. This chapter describes the ribonuclease protection assay, a widely accepted approach to evaluate the quality and amount of a target mRNA. This technique displays a higher sensitivity than classical Northern blot analysis and may be used either individually or in combination with other quantitative methods, such as quantitative reverse-transcription PCR (qRT-PCR), as complementary procedures rendering more complete and reliable information on gene expression.
\end{abstract}




\section{INTRODUCTION}

Gene expression control is a key point for cell development and survival across all living kingdoms, from bacteria to mammals. Since RNA molecules are the central effectors of the genetic information flow, the precise regulation of their relative abundance and stability is essential for the proper synthesis, assembly and localization of subcellular structures $(1,2)$. Regulation of the RNA levels is a multistep process that requires an accurate coordination between all the cellular processes. In eukaryotes, it begins in the nucleus, where protein factors specifically bind to DNA sequences activating or inhibiting the RNA synthesis. Nascent transcripts recruit diverse proteins that mediate multiple RNA modifications such as splicing, 5'-capping, 3'-polyadenilation and editing. Mature molecules are subsequently exported to the cytoplasm and directed to macromolecular complexes where they play essential roles in protein synthesis (rRNAs, mRNAs; (1)) and mRNA post-transcriptional regulation (miRNAs; $(3,4)$ ), among others. RNA molecules are eventually eliminated following diverse degradation pathways (57). In consequence, gene expression studies inevitably require the evaluation of the quality, stability and relative abundance of the intracellular RNA transcripts.

This chapter focuses on a classical strategy for the analysis of specific transcripts, the ribonuclease protection assay (RPA). It constitutes a very reliable and sensitive method for detection, quantification and analysis of RNA species in complex mixtures of total cellular RNA (8-11). RPA is based on the ribonucleases (RNases) ability to specifically discriminate between double and single stranded RNA templates. In this approach, RNA transcripts are hybridized in solution with a radioactive labelled RNA probe that is complementary to the molecule under investigation (Fig. 1). This interaction is performed under highly stringent conditions that are usually assessed by adding formamide to the hybridization buffer, which considerably reduces non-specific interactions. After hybridization, subsequent nuclease treatment removes non-interacting molecules. Several RNases specific for single-stranded RNA such as RNase T1 or RNase A can be used, either individually or in combination. Thus, non-hybridized RNA molecules or mis- 
matched RNA duplexes are degraded, while intact fully matched probe:transcript duplexes can be recovered by ethanol precipitation and resolved in denaturing polyacrylamide gels. With this technique, the size, amount and integrity of the desired RNA can be interrogated by subsequent exposure to autoradiography film or phosphoimaging (Fig. 2). Ideally, when this procedure is carried out with a molar excess of the probe over the target RNA, the intensity of the signal from the protected fragment is proportional to the amount of complementary RNA in the sample.

RPA is a widely accepted technique which complements the information obtained from qRT-PCR, with the advantage that it does not require a reverse transcription step. In addition, it provides additional data that cannot be obtained from qRT-PCR, like the size and integrity of a specific transcript. It may also substitute the Northern blotting analysis, in which the sensitivity, specificity and resolution limitations are often compromised by the inability to load large RNA amounts in the gel, inefficient transfer from gel to the blot membrane and cross or non-specific hybridization between probes and target RNA molecules. In addition, the hybridization in solution in the RPA favours the detection of rare transcripts. Furthermore, RPA replaces other classical nuclease-based methods, such as the S1 nuclease analysis, which usually employs DNA probes that are synthesized as double-stranded molecules. This entails that reconstitution of probe duplexes is probable, therefore reducing the sensitivity. Hence, careful purification of the probe strand is a requisite for a proper analysis.

RPA assays are well suited for mapping positions of external and internal junctions in RNA, such as transcription initiation and termination sites and intron/exon boundaries (12-14). With a proper probe design, RPA reveals the presence of sense and antisense transcripts from gene units (15) and allows for the estimation of genetic diversity in complex viral populations (16). It has been successfully used in the evaluation of the stability of therapeutic ribonucleoprotein complexes (17) and to quantify viral RNA replication levels (Fig. 2; (18)). RPA also allows to discriminate between closely related targets like members of a multigene family, by using probes designed to span the differing regions (19). These 
reactions can also be performed as multiplex assays to simultaneously analyze several transcripts (20), as long as the protected fragments exhibit different sizes. Moreover, its high sensitivity and specificity have been exploited for detection and quantification of microRNAs (miRNAs; (21)). Recently, modifications in the RPA technique have been reported to provide exclusive data that cannot be supplied by other methodologies. For instance, a sequential RPA/primer extension analysis has been described to distinguish among paralogous miRNAs that differ from each other by only one nucleotide located at their 5 ' end (22).

In summary, RPA is a reproducible and independent method that provides significant and valuable information in gene expression studies. In this chapter, we describe in detail the analysis of specific eukaryotic mRNAs by RPA analysis.

\section{MATERIALS}

All reagents and solutions, as well as plastic and glassware, must be free of ribonucleases. Gloves must be worn at all the times.

\subsection{RNA isolation}

1. Denaturing solution (see Note 1): $4 \mathrm{M}$ guanidinium thiocyanate; $25 \mathrm{mM}$ sodium citrate, $\mathrm{pH} 7.0 ; 0.5 \%$ (w/v) N-laurosylsarcosine (Sarkosyl); 0.1 M 2mercaptoethanol.

2. Sodium acetate $2 \mathrm{M}, \mathrm{pH} 4.0$.

3. Water-saturated phenol.

4. Chloroform:isoamyl alcohol (49:1, v/v) or bromochloropropane.

5. Isopropanol.

$6.80 \%$ ethanol.

7. RNase free sterile distilled water.

8. Glass-Teflon homogenizer. 


\subsection{Probe synthesis}

1. DNA templates (plasmid DNA or PCR products).

2. Restriction enzymes to digest the plasmid templates to define the proper $3^{\prime}$ end of the RNA.

3. NTP mix stock solution: $10 \mathrm{mM}$ adenosine 5 ' triphosphate (ATP), citosine $5^{\prime}$ triphosphate (CTP) and guanosine 5' triphosphate (GTP); $1 \mathrm{mM}$ uridine 5', triphosphate (UTP).

4. Transcription buffer, made according to the manufacturer's instructions. A common recipe is $40 \mathrm{mM}$ Tris- $\mathrm{ClH}, \mathrm{pH} 8.0 ; 6 \mathrm{mM} \mathrm{MgCl}_{2} ; 1 \mathrm{mM}$ spermidine; 4 $\mathrm{mM} \mathrm{NaCl} ; 10 \mathrm{mM}$ dithiothreitol; $0.01 \%$ Triton X-100 .

5. $\left[\alpha-{ }^{32} \mathrm{P}-\mathrm{UTP}\right], 10 \mathrm{mCi} / \mathrm{ml}, 800 \mathrm{Ci} / \mathrm{mmol}$. It is recommended to be used within 14 days of the labelling date to ensure high specific activity. It is a hazardous material, appropriate protection is required when handling.

6. Placental ribonuclease inhibitor $(40 \mathrm{U} / \mu \mathrm{l})$.

7. T7 RNA polymerase.

8. RNase-free DNase I (1-10 U/ $\mu$ l; e.g., RQ1 DNase from Promega).

9. $40 \%$ polyacrylamide stock solution (19:1 acrylamide:bis-acrylamide).

10. 10X TBE buffer: $0.89 \mathrm{M}$ Tris base; $0.89 \mathrm{M}$ boric acid; $25 \mathrm{mM}$ ethylenediamine-tetraacetic acid (EDTA).

11. Ammonium persulfate (APS) $10 \%(\mathrm{w} / \mathrm{v})$.

12. N, N, N', N'-tetramethyl-ethylene diamine (TEMED).

13. Formamide loading buffer: $97 \%$ formamide deionized ultrapure grade; 17 mM EDTA; $0.025 \%$ xylene cyanol; 0.025 bromophenol blue.

14. RNA elution buffer: $0.5 \mathrm{M}$ ammonium acetate; $0.1 \%$ sodium dodecyl sulphate (SDS); 1 mM EDTA.

15. Buffered saturated phenol.

16. Chloroform:isopropyl alcohol (24:1).

17. Sodium acetate 3M, pH 5.2 and pH 6.

18. $100 \%$ ethanol.

19. RNase free sterile distilled water. 
20. Sterile razor blades.

\subsection{Ribonuclease protection assay}

1. Hybridization buffer: $80 \%$ deionized formamide; $40 \mathrm{mM}$ PIPES, $\mathrm{pH} 6.4$; $400 \mathrm{mM} \mathrm{NaCl} ; 1 \mathrm{mM}$ EDTA.

2. Ribonuclease digestion buffer: $300 \mathrm{mM} \mathrm{NaCl} ; 10 \mathrm{mM}$ Tris- $\mathrm{HCl}, \mathrm{pH} 7.4 ; 5$ mM EDTA; 10 U RNase T1; $40 \mu \mathrm{g} / \mathrm{ml}$ RNase A.

3. SDS $20 \%(\mathrm{w} / \mathrm{v})$.

4. Proteinase K, $20 \mathrm{mg} / \mathrm{ml}$.

5. tRNA, $10 \mu \mathrm{g}$

6. Buffered saturated phenol.

7. Chloroform:isopropyl alcohol (24:1).

8. Sodium acetate $3 \mathrm{M}, \mathrm{pH} 5.2$ and $\mathrm{pH} 6$.

9. $100 \%$ ethanol.

10. Formamide loading buffer.

11. $40 \%$ polyacrylamide stock solution (19:1 acrylamide:bis-acrylamide).

12. TBE buffer.

11. APS $10 \%(\mathrm{w} / \mathrm{v})$.

12. TEMED.

12. RNase free sterile distilled water.

\section{METHODS}

\subsection{Total cellular RNA extraction}

The isolation of clean intact RNA is essential for the RPA. Procedures developed for extracting RNA from cultured cells involve complete cell lysis that liberates all the nucleic acids from different organelles. This involves that DNA and 
RNA are co-purified in the initial steps. Additional treatments are then required to remove the DNA. Other difficulty in RNA isolation is the contamination with intra- and extracellular ribonucleases, which are very stable and remain active under very suboptimal conditions. Hence, cell lysis in a chemical environment that inactivates nucleases is indispensable. Subsequent treatments allow for the selective purification of RNA from other macromolecular complexes.

There are several methods for preparing RNA from eukaryotic cells. Most of them employ common laboratory reagents, but commercial kits are also available. Here, we present a widely used protocol for total RNA extraction from adherent and suspension culture cells, the so-called guanidinium thiocyanatephenol-chloroform extraction method (23). It is based on the potent protein denaturing properties of the guanidinium thiocyanate. This chaotropic compound had been traditionally used as ribonuclease inhibitor by other authors in RNA extraction procedures $(24,25)$, but these required several ultracentrifugation steps to completely remove genomic DNA. This was notably time-consuming and limited the number of samples to be processed. The discovery that only total RNA remained soluble in the acidic aqueous phase after treatment with acid guanidinium thiocyanate-phenol-chloroform prompted the optimization of a faster purification protocol. The guanidinium thiocyanate-phenol-chloroform extraction method renders high yield of RNA from a small amount of start sample (tissue, cell culture), retrieves a good representation of the intracellular RNA pool and can be easily scaled up. The development of a single-step protocol was an important advance in the field and led to the marketing of kits that reduce hazardous handling and time for reagents preparation (RNAzol from MRC, TRI Reagent from Sigma-Aldrich or Trizol from Invitrogen-Life Technologies). The standard protocol for total RNA extraction is detailed below.

\section{Homogenization:}

For tissues: add $1 \mathrm{ml}$ of denaturing solution to $100 \mathrm{mg}$ of fresh tissue. Homogenize in a glass-Teflon homogenizer with a few strokes.

For culture cells: discard cell culture medium either by centrifugation (in case of cells growing in suspension) or by removing it from adherent cells. Wash cells 
twice in PBS. Then, add $1 \mathrm{ml}$ of denaturing solution per $10^{7}$ cells and mix by pipetting, which favours cell lysis and DNA fragmentation.

Samples must not remain in denaturing buffer for more than $30 \mathrm{~min}$.

2. Transfer the homogenate to a polypropylene tube.

3. Add 0.1 volumes of sodium acetate $2 \mathrm{M} \mathrm{pH} 4.0$ and mix thoroughly by inverting the tube.

4. One volume of water-saturated phenol is then supplied and mixed by inversion (see Note 2).

5. Extraction is completed by the addition of 0.2 volumes of chloroform:isoamyl alcohol (49:1) solution or bromochloropropane and vigorous shaking during $15 \mathrm{sec}$. This aids the dissociation of the nucleoprotein complexes.

6. Preserve the samples at room temperature during $3 \mathrm{~min}$.

7. Centrifugate $20 \mathrm{~min}$ at $10000 \mathrm{xg}, 4^{\circ} \mathrm{C}$.

8. The aqueous upper phase mostly containing the cellular RNA pool is carefully transferred to a new tube. RNA is then precipitated by adding one volume of $100 \%$ isopropanol and incubation at room temperature for $10 \mathrm{~min}$ (see Note 3). Centrifugate the samples at $10000 \mathrm{x} g$ during $20 \mathrm{~min}$ to pellet total RNA. It should appear as a gel-like precipitate.

9. Resuspend the precipitate in $1 \mathrm{ml}$ of ethanol $80 \%$ and vortex it to wash away the residual guanidinium. Incubate $10 \mathrm{~min}$ at room temperature.

10. Centrifugate $10 \mathrm{~min}$ at $10000 \mathrm{xg}, 4^{\circ} \mathrm{C}$. Discard the supernatant and air-dry the pellet.

11. Dissolve the RNA pellet in hybridization buffer by briefly vortexing or by heating at $60^{\circ} \mathrm{C}$ during $5 \mathrm{~min}$.

12. Spectrophotometric readings at wavelengths of 260 and $280 \mathrm{~nm}\left(\mathrm{~A}_{260}\right.$, $\mathrm{A}_{280}$ ), respectively, are required to analyze the RNA amount and the quality of the preparation. Measurements at $260 \mathrm{~nm}$ allow for the calculation of nucleic acid concentration, whereas the ratio $\mathrm{A}_{260} / \mathrm{A}_{280}$ estimates the contamination with phenol or proteins. This value should be between 1.8-2 in pure preparations. 


\subsection{Synthesis and purification of the RNA probes}

A DNA construct must be designed and produced for each specific RNA probe. The minimal requirement is a double-stranded promoter sequence upstream of the template for the desired RNA. The most common approach is the use of plasmid vectors carrying the T7 RNA polymerase promoter upstream of a multicloning site where the specific template sequences can be inserted. Alternatively, the use of PCR products as DNA templates carrying the T7 promoter sequence at their 5, end is also possible (see Note 4). Recommended probe size is 200 to 400 base pairs, though shorter probes may be used for abundant targets. Nevertheless, probe size is determined by the molecular feature to be sought. In any case, a careful removal of the DNA template must be accomplished to avoid the formation of heteroduplexes DNA template:RNA probe, which could promote misleading results. Probe gel purification is generally recommended, since incomplete transcripts may increase background levels and lost of sensitivity.

1. DNA template preparation:

For plasmid constructs:

Digest the plasmid containing the probe coding sequence with a restriction enzyme that cuts at the 3' end of the probe coding sequence. This provides a proper template for the RNA synthesis (Note 5).

For PCR templates:

Amplify the sequence of interest by PCR using a 5' primer containing the T7 RNA polymerase promoter sequence.

2. Check the quality of the template by agarose electrophoresis.

3. Extract DNA by adding an equal volume of buffered-saturated phenol. Mix well to form an emulsion and centrifugate for $2 \mathrm{~min}$ at $12000 \mathrm{x} g$.

4. Transfer the aqueous upper phase to a new tube and add one volume of chloroform:isoamyl alcohol (24:1). Mix by vortexing and centrifugate again.

5. Keep the upper phase and add 0.1 volume of $3 \mathrm{M}$ sodium acetate $\mathrm{pH} 6$ along with three volumes of cold $\left(-20^{\circ} \mathrm{C}\right)$ absolute ethanol.

6. Incubate at $-20^{\circ} \mathrm{C}$ for $30 \mathrm{~min}$ and centrifugate for $30 \mathrm{~min}$ at $12000 \times \mathrm{g}, 4^{\circ} \mathrm{C}$. 
7. Discard the supernatant and wash the pellet with $300 \mu \mathrm{l}$ of $70 \%$ ethanol.

8. Centrifugate for $5 \mathrm{~min}$ at $12000 \mathrm{xg}, 4^{\circ} \mathrm{C}$.

9. Remove the supernatant and vacuum-dry briefly the DNA pellet.

10. Dissolve the template in RNase free sterile distilled water.

11. Set up $50 \mu \mathrm{l}$ transcription reactions in transcription buffer $1 \mathrm{X}$ containing 1 $\mu \mathrm{g}$ of purified DNA template, $5 \mu \mathrm{Ci}$ of $\left[\alpha-{ }^{32} \mathrm{P}\right] \mathrm{UTP}(3000 \mathrm{Ci} / \mathrm{mmol}), 1 \mathrm{mM} \mathrm{GTP}$, $1 \mathrm{mM}$ ATP, $1 \mathrm{mM} \mathrm{CTP}, 0.1 \mathrm{mM}$ UTP (to achieve high specific activity of the RNA probe; Note 6), $0.5 \mathrm{U} / \mu \mathrm{l}$ of placental ribonuclease inhibitor and $20 \mu \mathrm{g} / \mathrm{ml}$ of purified T7 RNA polymerase.

12. Incubate the reaction mix at $37^{\circ} \mathrm{C}$ for $2 \mathrm{~h}$.

13. Stop the reaction by adding $1 \mathrm{U}$ of RNase-free DNase and incubate at $37^{\circ} \mathrm{C}$ for $15 \mathrm{~min}$.

14. An equal volume of formamide loading buffer $(50 \mu \mathrm{l})$ is added and samples are heated at $95{ }^{\circ} \mathrm{C}$ in a dry bath before loading directly on preparative denaturing polyacrylamide-7 $\mathrm{M}$ urea gels. The gel concentration should be chosen according to the size of the RNA molecule to be purified (3.5\% - $20 \%$ ). Electrophoresis should be performed under denaturing conditions in TBE $0.5 \mathrm{X}$.

15. Cover the gel with a saran-wrap and place it on an exposure cassette with a sheet of X-ray film. Expose it in a dark room (see Note 7). The acrylamide slice containing the full-length probe is excised using a razor blade.

16. The gel slices are then soaked in $300 \mu \mathrm{l}$ of RNA elution buffer and incubated overnight at $4{ }^{\circ} \mathrm{C}$.

17. Purify the RNA by consecutive phenol and chloroform:isoamyl alcohol extraction as indicated in steps 3-5.

18. Precipitate the RNA by adding 0.1 volume of 3 M sodium acetate $\mathrm{pH} 5.2$ and three volumes of cold $\left(-20^{\circ} \mathrm{C}\right)$ absolute ethanol.

19. Pellet the RNA as noted in steps 6-9.

20. Dissolve the labelled RNA in hybridization buffer.

21 . Check the specific activity of the probe in a scintillation counter. The product should be stored at $-20^{\circ} \mathrm{C}$ until use. 


\subsection{Ribonuclease protection assay}

1. Mix $10 \mu \mathrm{g}$ of total cellular RNA with $10^{5} \mathrm{cpm}$ of RNA probe in $30 \mu \mathrm{l}$ of hybridization buffer (see Note 8). Include a negative control with tRNA instead of total RNA. This provides information about the background hybridization and the yield of the ribonuclease digestion reaction (see Note 9).

2. Denature RNA by heating at $85^{\circ} \mathrm{C}$ during $5 \mathrm{~min}$.

3. Immediately transfer the mix to the hybridization temperature (see Note 10).

4. Anneal overnight.

5. Add $300 \mu \mathrm{l}$ of RNase digestion buffer and incubate at $30^{\circ} \mathrm{C}$ for 30 to $60 \mathrm{~min}$.

6. Stop the reaction by the addition of SDS $1 \%$ and $10 \mathrm{mg} / \mathrm{ml}$ of proteinase $\mathrm{K}$. Incubate $30 \mathrm{~min}$ at $37^{\circ} \mathrm{C}$.

7. Undigested RNA is extracted with phenol:chloroform:isoamyl alcohol and precipitated with ethanol as described above.

8. Dry pellets are resuspended in formamide loading buffer and resolved by denaturing polyacrylamide-urea gels.

\subsection{Analysis}

Gels are exposed to autoradiography film or phosphoimaging and subsequently analyzed and quantified by densitometric or fluorescence scanning.

One of the main RPA applications is the absolute quantification of a specific RNA. For that purpose, a standard curve must be established using known amounts of the in vitro transcribed target hybridized with an excess of the probe. After ribonuclease treatment, products are resolved in denaturing polyacrylamide gels. The intensity of the signal is compared to that generated by the experimental samples and used to determine the absolute amount of target RNA. 


\section{NOTES}

1. Prepare a $7 \mathrm{M}$ guanidinium thiocyanate stock solution in RNase free sterile distilled water by heating at $65^{\circ} \mathrm{C}$. Then, add sodium citrate $(\mathrm{pH} 7.0)$ to reach a final concentration of $45 \mathrm{mM}$ and $26.4 \mathrm{ml}$ of $10 \%(\mathrm{w} / \mathrm{v})$ Sarkosyl $(N-$ laurosylsarcosine). The stock solution can be stored up to 3 months at room temperature. To prepare the working denaturing solution, just add 2-mercaptoethanol ( $0.5 \%$ final concentration). This solution can be stored up for 1 month at room temperature. To minimize handling of guanidinium thiocyanate, dissolve it directly in the manufacturer's bottle. The 2-mercaptoethanol should be handled under a fume hood.

2. Preservation of the acidic $\mathrm{pH}$ is a key factor in the success of the RNA isolation method. A low $\mathrm{pH}$ value ensures the proper separation of RNA from DNA and proteins. For this reason, it is recommended the use of water-saturated phenol instead of buffered-saturated phenol.

3. RNA precipitation can be improved by increasing the incubation time up to 1 h. In this case, samples should be maintained at $-20^{\circ} \mathrm{C}$. This is specially recommended when working with low amount of starting material.

4. T7 RNA polymerase efficiently synthesizes RNA molecules in vitro with minimal requirements. These include the $17 \mathrm{nt}$ double-stranded promoter sequence followed by GGG and a single-stranded template.

\section{5' - TAATACGACTCACTATA $\downarrow$ GGG 3' - ATTATGCTGAGTGATAT CCCXXXX.........XXX - 5',}

Structure of the partially double-stranded DNA template.

T7 promoter sequence is shown in bold; $\downarrow$ indicates the transcription initiation site.

5. The enzyme may cut the plasmid in multiple positions, as long as it does not cut into the promoter or the probe sequence. Complete digestion of the plasmid template should be checked by agarose electrophoresis. The presence of non- 
digested molecules in the reaction results in run on transcripts that use up nucleotides and reduce the yield of the probe synthesis.

6. This is a general protocol that must be optimized for each specific probe. The nucleotide concentration is especially relevant: the lower amount of nucleotide, the higher proportion of incomplete transcripts. For short RNA probes (less than $400 \mathrm{nt}$ ), $1 \mathrm{mM}$ of each non-labelled NTP generally renders high transcription yields. Long probes require specific transcription buffers with higher amounts of nucleotides and divalent cations. In many cases, it may be necessary the addition of non-radioactive limiting nucleotides to preserve the nucleotide concentration high enough to produce significant amounts of full-length transcript. This leads to a decreased specific activity that will be compensated by the increased sensitivity of the longer probe. Alternatively, commercial kits are currently available that produce high amounts of long RNA molecules (up to $3000 \mathrm{nt}$ ).

7. The position of the film on the gel is recorded using a marker, with nonsymmetric lines drawn across all edges of the film to ensure its correct repositioning on top of the gel after development. Alternatively, a variety of phosphorescent labels or markers available from different commercial sources can be used to guarantee the correct alignment of the film on the gel. A 2-5 minutes exposure should be enough for efficient transcription when using nucleotides with high specific activity.

8. For total cellular RNA, $10 \mu \mathrm{g}$ is enough to detect most of RNA molecules. However, this amount can be increased for low abundant species.

9. Background hybridization is commonly due to incomplete digestion of the DNA template after the probe transcription reaction. Residual DNA template molecules can efficiently interact with the probe, leading to heteroduplexes DNA:RNA that are not cleaved by ribonucleases. Background can be also caused by the presence of trace amounts of non-digested single-stranded probe. In both cases, this generates smears that appear in all the reactions. A tRNA negative control allows for the evaluation of the background.

10. There is an optimal annealing temperature for each probe, which depends not only on its sequence but also on its secondary structure. It is therefore advis- 
able to experimentally test the optimal temperature by using a range from $30^{\circ} \mathrm{C}$ to $60{ }^{\circ} \mathrm{C}$.

\section{Acknowledgments}

A.B.-H's group is funded by grant BFU2009-08137 from the Spanish Ministry of Science and Innovation, grant CTS-5077 from the Junta de Andalucía, and by FEDER funds from the EU. P.M.'s group is supported by the Andalusian Health Department, Andalusian Innovation and Science Department (P08-CTS-3678 to P.M), the FIS (PI070026), the MINICC (PLE-2009-0111) and the Marie Curie (PIIF-GA-2009-236430). We are indebted to Dr. Saumitra Das for his kind gift of figure 2 .

\section{REFERENCES}

1. Moore, M. J. (2005) From birth to death: the complex lives of eukaryotic mRNAs. Science 309, 1514-1518.

2. Halbeisen, R. E., Galgano, A., Scherrer, T., and Gerber, A. P. (2008) Post-transcriptional gene regulation: from genome-wide studies to principles. Cell Mol Life Sci 65, 798-813.

3. Eulalio, A., Huntzinger, E., and Izaurralde, E. (2008) Getting to the root of miRNA-mediated gene silencing. Cell 132, 9-14.

4. Chekulaeva, M., and Filipowicz, W. (2009) Mechanisms of miRNA-mediated posttranscriptional regulation in animal cells. Curr Opin Cell Biol 21, 452-460.

5. Fritz, D. T., Bergman, N., Kilpatrick, W. J., Wilusz, C. J., and Wilusz, J. (2004) Messenger RNA decay in mammalian cells: the exonuclease perspective. Cell Biochem Biophys 41, 265 278.

6. Parker, R., and Song, H. (2004) The enzymes and control of eukaryotic mRNA turnover. Nat Struct Mol Biol 11, 121-127.

7. Schmid, M., and Jensen, T. H. (2008) The exosome: a multipurpose RNA-decay machine. Trends Biochem Sci 33, 501-510.

8. Zinn, K., DiMaio, D., and Maniatis, T. (1983) Identification of two distinct regulatory regions adjacent to the human beta-interferon gene. Cell 34, 865-879.

9. Melton, D. A., Krieg, P. A., Rebagliati, M. R., Maniatis, T., Zinn, K., and Green, M. R. (1984) Efficient in vitro synthesis of biologically active RNA and RNA hybridization probes from plasmids containing a bacteriophage SP6 promoter. Nucleic Acids Res 12, 7035-7056.

10. Winter, E., Yamamoto, F., Almoguera, C., and Perucho, M. (1985) A method to detect and characterize point mutations in transcribed genes: amplification and overexpression of the mutant c-Ki-ras allele in human tumor cells. Proc Natl Acad Sci U S A 82, 7575-7579.

11. Lee, J. J., and Costlow, N. A. (1987) A molecular titration assay to measure transcript prevalence levels. Methods Enzymol 152, 633-648.

12. Burczynski, M. E., Lin, H. K., and Penning, T. M. (1999) Isoform-specific induction of a human aldo-keto reductase by polycyclic aromatic hydrocarbons (PAHs), electrophiles, and oxidative stress: implications for the alternative pathway of PAH activation catalyzed by human dihydrodiol dehydrogenase. Cancer Res 59, 607-614.

13. Benkusky, N. A., Fergus, D. J., Zucchero, T. M., and England, S. K. (2000) Regulation of the $\mathrm{Ca} 2+$-sensitive domains of the maxi-K channel in the mouse myometrium during gestation. $J$ Biol Chem 275, 27712-27719. 
14. Stabell, F. B., Tourasse, N. J., Ravnum, S., and Kolsto, A. B. (2007) Group II intron in Bacillus cereus has an unusual 3 ' extension and splices 56 nucleotides downstream of the predicted site. Nucleic Acids Res 35, 1612-1623.

15. Chen, G. L., and Miller, G. M. (2009) 5'-Untranslated region of the tryptophan hydroxylase-2 gene harbors an asymmetric bidirectional promoter but not internal ribosome entry site in vitro. Gene 435, 53-62.

16. Cabrera, O., Roossinck, M. J., and Scholthof, K. B. (2000) Genetic Diversity of Panicum mosaic virus Satellite RNAs in St. Augustinegrass. Phytopathology 90, 977-980.

17. Kim, J., Lee, S. H., Choe, J., and Park, T. G. (2009) Intracellular small interfering RNA delivery using genetically engineered double-stranded RNA binding protein domain. J Gene Med 11, 804-812.

18. Ray, P. S., and Das, S. (2004) Inhibition of hepatitis C virus IRES-mediated translation by small RNAs analogous to stem-loop structures of the 5'-untranslated region. Nucleic Acids Res 32, 1678-1687.

19. Brown, P. C., Thorgeirsson, S. S., and Silverman, J. A. (1993) Cloning and regulation of the rat mdr2 gene. Nucleic Acids Res 21, 3885-3891.

20. Cloutier, N., Gravel, A., and Flamand, L. (2004) Multiplex detection and quantitation of latent and lytic transcripts of human herpesvirus-8 using RNase Protection Assay. J Virol Methods 122, 1-7.

21. Lee, Y., Jeon, K., Lee, J. T., Kim, S., and Kim, V. N. (2002) MicroRNA maturation: stepwise processing and subcellular localization. Embo $J$ 21, 4663-4670.

22. Zeiner, G. M., and Boothroyd, J. C. Use of two novel approaches to discriminate between closely related host microRNAs that are manipulated by Toxoplasma gondii during infection. Rna 16, 1268-1274.

23. Chomczynski, P., and Sacchi, N. (1987) Single-step method of RNA isolation by acid guanidinium thiocyanate-phenol-chloroform extraction. Anal Biochem 162, 156-159.

24. Chirgwin, J. M., Przybyla, A. E., MacDonald, R. J., and Rutter, W. J. (1979) Isolation of biologically active ribonucleic acid from sources enriched in ribonuclease. Biochemistry 18, 5294-5299.

25. Katoch, V. M., and Cox, R. A. (1986) Step-wise isolation of RNA and DNA from mycobacteria. Int J Lepr Other Mycobact Dis 54, 409-415.

\section{FIGURE LEGENDS}

Fig. 1 Cartoon describing the ribonuclease protection assay (RPA). Purified RNA samples are hybridized in solution with a molar excess of a radioactive labelled RNA probe to generate RNA duplexes. Unpaired RNA molecules are digested by the ribonuclease cocktail and protected fragments are resolved in denaturing polyacrylamide gels. These are subsequently exposed to evaluate the size and amount of the RNA under investigation

Fig. 2 Representative ribonuclease probing assay (RPA) for relative quantification of viral RNA. A human derived hepatoma cell line containing a replicon system for the hepatitis $\mathrm{C}$ virus (HCV) was transfected with different amounts of the 
HCV RNA domain SLIIIe+f. Total cellular RNA was hybridized overnight with $10000 \mathrm{cpm}$ of a sense RNA probe for the 5' end of the HCV genome. After ribonuclease digestion, products were resolved on $10 \%$ denaturing polyacrylamide gels. Lane M denotes molecular weight marker. Figure is a kind gift of Dr. Saumitra Das (18) by permission of Oxford University Press 